\title{
Systemic-onset juvenile rheumatoid arthritis and ANCA-associated glomerulonephritis
}

\author{
A Belot*1, B Bader-Meunier ${ }^{2}$, LH Noel², AM Prieur ${ }^{2}$, P Niaudet $^{2}$, R Salomon ${ }^{2}$ \\ and P Quartier ${ }^{2}$
}

Address: ${ }^{1}$ Hopital Femme Mère Enfant, Lyon, France and ${ }^{2}$ Hopital Necker-Enfants Malades, Paris, France

* Corresponding author

from $15^{\text {th }}$ Paediatric Rheumatology European Society (PreS) Congress

London, UK. 14-17 September 2008

Published: 15 September 2008

Pediatric Rheumatology 2008, 6(Suppl I):P25 doi:I0.I I86/I546-0096-6-SI-P25

This abstract is available from: http://www.ped-rheum.com/content/6/SI/P25

(C) 2008 Belot et al; licensee BioMed Central Ltd.

Systemic-onset juvenile rheumatoid arthritis (SoJRA) associated renal lesions are unusual, except for amyloidosis or side effects of antirheumatic drugs.

We report on 3 children with SoJRA diagnosed in the first years of life, who presented a perinuclear antineutrophil cytoplasmic antibodies (pANCA) associated glomerulonephritis during the disease course.

ANCA glomerulonephritis was diagnosed after 5, 6 and 1 year follow-up for SoJRA in Patients 1, 2 and 3 respectively.

Treatment and outcome of the kidney lesions were as follows:

Pt1 was treated with steroids bolus associated to rituximab then switched for mycophenolate mofetyl (MMF). At the age of 7, he benefited from anakinra for the still ongoing systemic symptoms of the SoJRA with excellent results on both inflammation baseline and proteinuria.

Despite steroids bolus and azathioprine in Pt2, renal lesions led gradually to end stage renal disease (ESRD) when she was 17 . She benefited from kidney transplantation and interestingly both systemic symptoms and renal function are well under triple immunosuppressant (MMF, tacrolimus and steroids) with a follow up of 3 years after transplantation.
Pt3 evolved to ESRD despite the use of steroids bolus, cyclophosphamide, cyclosporine and immunoglobulin. She died when she was 16 years, after 4 years of dialysis, from a severe sepsis with digestive occlusion.

ANCA vasculitis is exceptional in the JRA. Outcome is poor and can lead to ESRD and death. ANCA-associated glomerulonephritis were related to SoJRA with uncontrolled inflammation baseline. These data suggest a common pathogenesis between SoJRA and ANCA glomerulonephritis and underline overlapping syndrome in the pediatric practice. 